
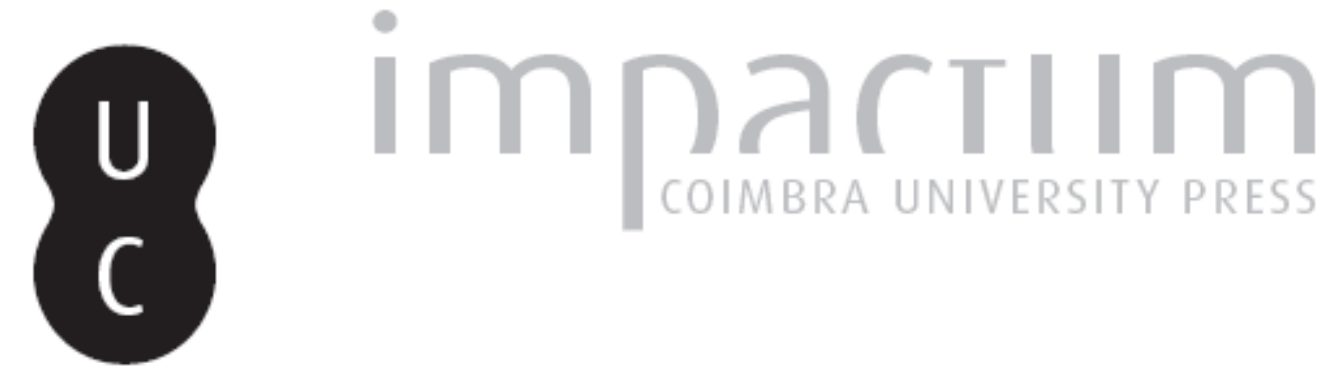

\title{
La propagation du béton armé au Portugal: les bâtiments industriels comme moyens de divulgation de la technique
}

Autor(es): Licordari, Mariangela

Publicado por: Imprensa da Universidade de Coimbra

URL persistente:

URI:http://hdl.handle.net/10316.2/40964

DOI:

DOI:https://doi.org/10.14195/1645-2259_16_14

Accessed : $\quad$ 26-Apr-2023 13:15:30

A navegação consulta e descarregamento dos títulos inseridos nas Bibliotecas Digitais UC Digitalis, UC Pombalina e UC Impactum, pressupõem a aceitação plena e sem reservas dos Termos e Condições de Uso destas Bibliotecas Digitais, disponíveis em https://digitalis.uc.pt/pt-pt/termos.

Conforme exposto nos referidos Termos e Condições de Uso, o descarregamento de títulos de acesso restrito requer uma licença válida de autorização devendo o utilizador aceder ao(s) documento(s) a partir de um endereço de IP da instituição detentora da supramencionada licença.

Ao utilizador é apenas permitido o descarregamento para uso pessoal, pelo que o emprego do(s) título(s) descarregado(s) para outro fim, designadamente comercial, carece de autorização do respetivo autor ou editor da obra.

Na medida em que todas as obras da UC Digitalis se encontram protegidas pelo Código do Direito de Autor e Direitos Conexos e demais legislação aplicável, toda a cópia, parcial ou total, deste documento, nos casos em que é legalmente admitida, deverá conter ou fazer-se acompanhar por este aviso.

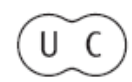




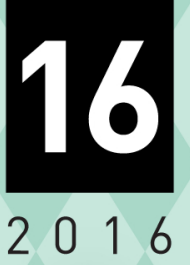

\section{Revista \\ de História \\ da Sociedade \\ e da \\ Cultura}




\title{
La propagation du béton armé au Portugal: les bâtiments industriels comme moyens de divulgation de la technique
}

\section{The diffusion of reinforced concrete in Portugal: industrial buildings as means of technical disclosure}

\author{
MARIANGELA LICORDARI \\ $\mathrm{PhD}$ Histoire de l'Art (ED 441) \\ Univ. Paris 1 Panthéon-Sorbonne; IHA-UNL \\ mariangela.licordari@gmail.com
}

Texto recebido em / Text submitted on: 29.02.2016

Texto aprovado em / Text approved on: 30.06.2016

Résumé: Cet article vise à donner des précisions sur la propagation du béton armé au Portugal, en cherchant à clarifier les mécanismes et les coïncidences qui ont permis, au tournant du $\mathrm{XX}^{\mathrm{e}}$ siècle, la divulgation de cette nouvelle technique de construction dans ce pays au début de son industrialisation. Pour la diffusion de ce nouveau matériau dans le contexte constructif du Portugal, les compétences entrepreneuriales de François Hennebique, père du brevet en béton armé le plus connu à l'échelle internationale, seront fondamentales. Les bâtiments industriels seront les premiers édifices capables d'exploiter au maximum les qualités techniques du nouveau matériau. La Fábrica de Moagem de Caramujo est l'un des exemples les plus concrets pour justifier notre argumentaire. Ce bâtiment a été le premier au Portugal à être entièrement construit en béton armé, en laissant comme héritage un lexique constructif qui trouvera une utilisation très large dans l'architecture portugaise des années à venir.

Mots clés: béton armé, $\mathrm{XX}^{\mathrm{e}}$ siècle, Portugal, bâtiments industriels, architecture.

Abstract: This article aims to provide information on the spread of reinforced concrete in Portugal, seeking in particular to clarify the mechanisms, the coincidences and key people who allowed, at the turn of the $20^{\text {th }}$ century, the disclosure of this new construction technique in a country beginning its industrialization. For the dissemination of this new material in the constructive context of Portugal the entrepreneurial skills of François Hennebique, creator of the best world known patent in reinforced concrete, will be fundamental. The industrial buildings will be the first able to able to maximize the technical qualities of the new material. The Fábrica de Moagem de Caramujo represents the most tangible example of what has been said. This building was the first in Portugal to be entirely built in reinforced concrete, leaving as a legacy a constructive lexicon that will find a wide use in future modern Portuguese architecture.

Keywords: reinforced concrete, $20^{\text {th }}$ century, Portugal, industrial buildings, architecture. 


\section{Introduction}

Connaître le processus de diffusion du béton armé au Portugal est d'un intérêt particulier surtout si on le considère en étroite relation avec l'évolution formelle de l'architecture portugaise du début du $\mathrm{XX}^{\mathrm{e}}$ siècle et avec les répercussions stylistiques de l'architecture contemporaine des années 70-90. La caractéristique de la "synthèse architecturale" portugaise, qui dans certains architectes contemporains tels que Álvaro Siza et Eduardo Souto de Moura atteindra tons poétiques, part en fait de racines profondes liées au passé qui trouvent leur origine dans les innovations techniques et esthétiques du béton armé. L'architecture portugaise moderne, en particulier à partir des années 50, doit être comprise à la lumière d'une architecture créée par le mélange de deux cultures: l'une traditionnelle et l'autre, innovante, du béton armé. Au Portugal, la compréhension de la transition de l'architecture traditionnelle à l'architecture moderne passe inévitablement, non seulement à la compréhension de l'histoire de la propagation du béton armé, nouveau matériau de construction pour le pays, mais également au rôle prépondérant qu'il a su jouer dans les bâtiments industriels et dans les différents secteurs de l'industrie de la construction. Comprendre cela fait reconnaitre l'importance qu'une technique constructive a dans le bouleversement architecturale d'une idée de projet et par conséquence l'importance de la connaissance de son histoire de propagation.

\section{Contexte général}

$\mathrm{Au}$ début du $\mathrm{XX}^{\mathrm{e}}$ siècle, au niveau international, l'environnement économique, culturel et sociologique lié au monde du bâtiment est prêt de façon plus concrète à l'expérimentation d'un nouveau matériau: le béton armé. L'activité industrielle au tournant du siècle, caractérisée par une réalité sociale plus dynamique et ouverte à l'introduction de nouvelles technologies, déterminera la divulgation de ce matériau surtout dans un domaine qui sans plus tarder favorisera la révolution esthétique et formelle de l'architecture du $\mathrm{XX}^{\mathrm{e}}$ siècle: celui des établissements industriels (Licordari 2013: 70-74).

Les historiens de l'architecture qui s'intéressent au premier âge du béton armé, ont parfois tendance à commenter seulement les ouvrages les plus originaux et marginaux de la production de ce nouveau matériau, comme la maison que Ward a édifié en 1873 à Port Chester ou l'église Saint-Jean de Montmartre de De Baudot et Cottancin, qui est toujours un ouvrage 
hors genre, sans antécédent tant par le style que par la technique (Simonnet 2005: 73). Toutefois, il faut reconnaître que les premiers bâtiments qui constituaient les premiers ballons d'essai du béton armé ont été des bâtiments utilitaires. En particulier, dans les bâtiments industriels au tournant du XIX ${ }^{\mathrm{e}}$ siècle et au début du $\mathrm{XX}^{\mathrm{e}}$, l'exploitation de cette nouvelle technique constructive sera déterminée soit par la nécessité d'un espace de travail approprié aux nouvelles exigences productives, pour lesquelles concevoir une disposition précise des lieux en fonction du volume des machineries et en fonction des flux, des déplacements et des conditions de stockage des matériaux et des produits, soit par la nécessité de résoudre de façon définitive le problème principal de plusieurs bâtiments industriels: le feu. Par exemple, ce n'était pas un hasard que, pour la divulgation de son célèbre brevet de 1892, François Hennebique (1842-1921) choisissait de publier sa brochure propagandiste sous le titre Plus d'incendies désastreux. C'était exactement dans le but d'attirer l'attention de la plupart des entreprises de construction, qui deviendront par la suite ses concessionnaires, et des capitaines d'industrie propriétaires des usines. Un autre moyen de propagande de l'entrepreneur belge était sa revue Le Béton armé (1898), où Hennebique avait l'habitude d'éditer sous forme d'inventaire la liste de toutes ses réalisations. Il est à travers cette liste qu'on connait les premières expérimentations de la firme dans le monde. La quasi-totalité des affaires recensées dans ces relevés sont d'origine privée et se partagent entre construction ou équipement industriel et habitation. A partir de 1897, la quantité d'affaires relatées ( 714 seulement en 1898) oblige Hennebique à diviser en familles d'ouvrages la longue liste qu'il publie. La division prévoyait une classification en habitations (199 affaires), constructions religieuses (35), bâtiments publics (68), constructions industrielles (169), bâtiments à usage de magasins (59), ponts et réservoirs (85) et divers fondations, canalisations, etc (99). En réalité, la plupart de ces programmes, dont le chiffre va s'amplifiant d'année en année, concernent encore une fois des planchers et des renforcements de structures, à l'exception de certains exemples qui comprennent la totalité de l'ossature, comme par exemple 168 constructions industrielles et 60 entrepôts et magasins réalisés en 1898 (Simonnet 2005: 74).

Dans le cas spécifique de l'architecture industrielle, donc, l'avènement du béton armé constituait un tournant majeur dans la façon de concevoir. Dès le début, la nouvelle manière de "faire l'architecture" attache une grande importance à la notion d'espaces ouverts diffusément éclairés. Ceci était possible grâce au système de construction appelé à ossature qui consistait en un squelette de poutres et de poteaux en béton armé complété 
par des ponts horizontaux eux-mêmes en béton (brevet Hennebique). Le nouveau matériau devient tout de suite synonyme de grands espaces et de grandes ouvertures vitrées. Par rapport aux techniques constructives traditionnelles, le béton armé présente certains avantages clairs, parmi lesquels une augmentation significative de la capacité portante, une résistance au feu plus élevée, une réduction significative de la hauteur des planchers et de l'épaisseur des parois et enfin de meilleures possibilités d'organisation de l'espace intérieur, grâce à la notion de plan libre. Aussi le périmètre de murs porteurs se transforme-il considérablement par l'insertion d'éléments verticaux et horizontaux, en créant une nouvelle conception architecturale de la façade (façade libre).

Le terrain d'actions du nouveau matériau est lié tant aux nouveaux secteurs de l'industrie lourde qu'à ceux déjà existants de l'industrie traditionnelle. A cette grande industrie, s'ajoutent une série de petites industries, comme les usines pour la production de la farine, les moulins, les papeteries, les usines de chaussures, etc., qui deviennent eux aussi les protagonistes de cette première phase de diffusion du béton armé, et un ensemble d'autres constructions du monde industriel tels que les silos, les magasins, les réservoirs...

Il revient à Monier d'expérimenter les premières utilisations du béton dans la construction industrielle, à travers la réalisation à Sèvres-aux-Bruyeres de deux réservoirs pour la Société Générale des Eaux (1872), des constructions de 16 mètres de diamètre chacun. Ces ouvrages seront la première application documentée de son système, même s'il faut reconnaître que jusquà ce moment-là l'invention de Monier ne pouvait pas encore être considérée le "béton armé" d'aujourd'hui. Il s'agissait plutôt de fer-ciment qui seulement en 1886, après les élaborations de Matthias Koenen, pourra véritablement être appelé béton armé, étant utilisé pour la réalisation d'un système de construction pour des maisons, fixes ou portatives, hygiéniques et économiques en ciment et fer (Iori 2001: 16).

Mais il sera donné à Hennebique la tâche de diffuser le nouveau matériau dans les établissements industriels de la période, conquérant ainsi un marché de la construction encore mal défini par les autres maisons rivales. Cela a favorisé le début d'exploitation de son brevet, surtout au niveau d'un aspect technique très important dans la conception d'espace de l'usine qui est l'expérimentation des éléments structurels linéaires (poutres et piliers) qui constituent le nœud de son système et qui ont jeté les bases pour l'affirmation de la "structure renforcée encadrée" en béton armé indépendante de la maçonnerie. Parmi tous les brevets du béton armé, le brevet Hennebique doit être reconnu comme le brevet par excellence, capable de répandre 
le nouveau matériau dans presque tous les domaines de la construction. Il s'agissait d'un brevet tout à faite rationnel qui permettait la construction complète d'un squelette monolithique en béton armé. Les éléments structuraux de cette ossature révolutionnaire en béton, qui sera enregistrée avec le brevet du 1892, étaient: le pilier, avec ses caractéristiques bords arrondis et ses dimensions généralement de petite taille $(40 \times 40 \mathrm{~cm}, 50 \times 50 \mathrm{~cm} \ldots)$; les poutres principales décrochées du plafond avec des bords arrondis et reliées aux colonnes avec des crochets; les poutres secondaires qui rigidifient les dalles et s'insèrent dans les poutres principales, et les dalles de faible épaisseur de forme rectangulaire ou carrée. Ces éléments vont constituer le cœur du système constructif cartésien qui deviendra crucial dans la gestion de l'espace du travail de l'usine, en déterminant une évolution du concept d'usine en manière moderne.

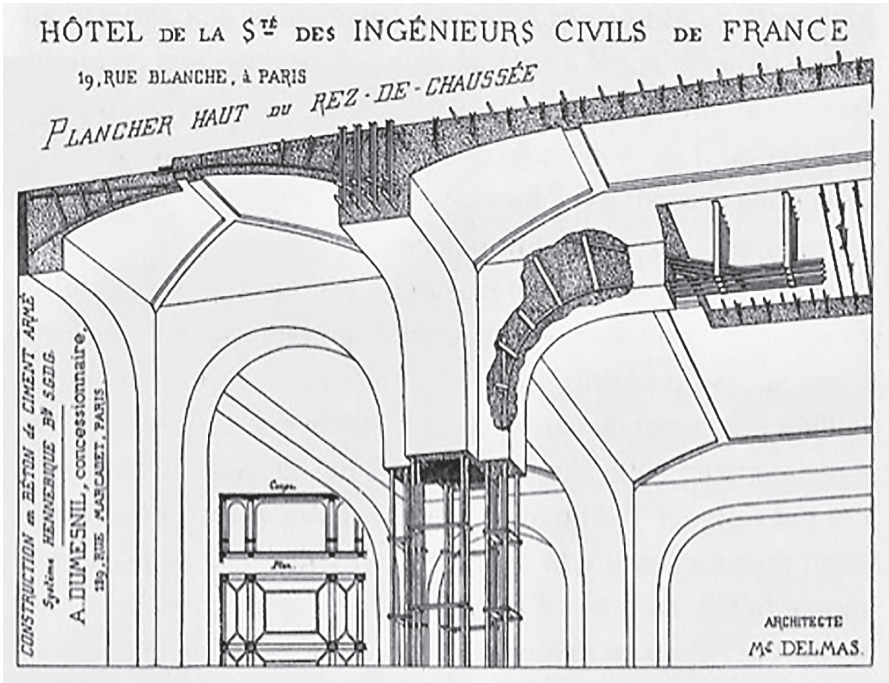

Fig. 1.

Logo entreprise Hennebique qui montre la liaison structurale poutre-poteau (Delhumeau 1999: 242)

Ces reconnues qualités structurelles, hygiéniques et fonctionnelles détermineront sa propagation rapide dans le domaine de la construction de tous les pays industrialisés, avec bien sûr des différences appropriées d'adoption et d'adaptation liées à la nature économique et sociologique des différents pays de diffusion. Dans le cas du Portugal, par exemple, les conditions sociales et économiques du pays au tournant du $\mathrm{XX}^{\mathrm{e}}$ siècle et sa modernisation de production rapide ont été fondamentales à la pleine affirmation du béton armé en général et du brevet Hennebique en particulier; en outre, la connaissance séculaire que les portugais avaient des "structures encadrées" - prenons 


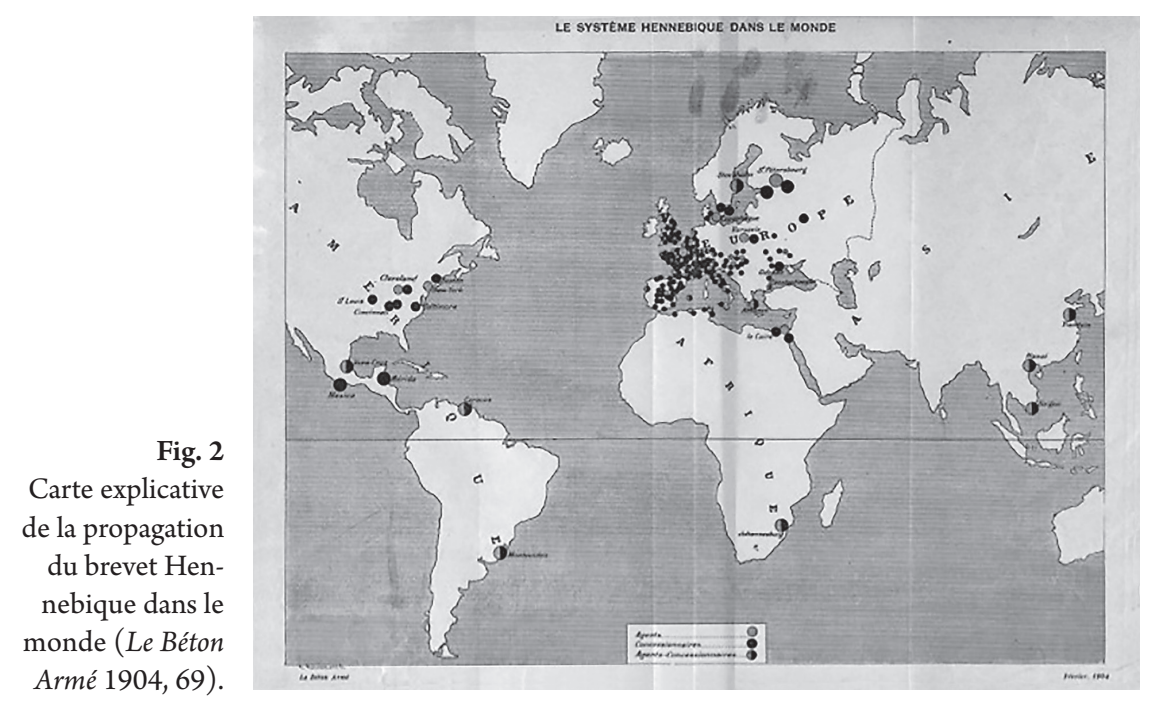

l'exemple de la célèbre gaiola pombalina ${ }^{1}$, ossature en bois incorporée dans la maçonnerie et utilisée à Lisbonne comme système antisismique après le tremblement de terre de 1755 - a probablement permis une acceptation plus rapide du système de construction.

\section{Le béton armé au Portugal : la parenthèse du brevet Hennebique}

Au Portugal, aussi bien que dans les autres pays européens, l'entrée du béton armé dans le groupe des matériaux de construction s'est effectuée à la mesure de l'audace et de l'action innovatrice de certains personnages, tels que auteurs de projets ou constructeurs, qui avec enthousiasme avaient adhéré au nouveau matériau. Étant un matériau nouveau, qui n'était pas lié à l'histoire de la construction moderne portugaise comme ce fut pour le cas de la pierre, la brique et le bois, le béton armé s'introduit au niveau de la construction portugaise par la voie d'objets considérés habituellement comme les moins représentatifs d'un point de vue culturel, comme les entrepôts et magasins, les silos et quais portuaires, les ponts, les usines et ateliers qui à cette époque là deviennent le milieu expérimental et le meilleur véhicule d'introduction du matériau dans le complexe urbain.

1 À cet égard, voir la nombreuse littérature sur le sujet, tels que Farinha 1997. 
Comme l'on a déjà mentionné, la diffusion du béton armé au Portugal est liée à la croissance industrielle que le pays était en train de vivre au tournant du siècle et à la régénération urbaine que cette croissance imposait. Au début $\mathrm{du} \mathrm{XX}^{\mathrm{e}}$ siècle la culture portugaise se débattait entre un désir de modernisation, qui reposait sur la croyance optimiste vers le potentiel de la machine, et une nostalgie vers le passé qui méprisait ce présent caractérisé par cette mutation accélérée. Le monde de la construction en quelque sorte reflétait la dichotomie de cette période de transition, où les valeurs artistiques de l'architecture du passé venaient de plus en plus confrontées avec l'efficacité de l'ingénierie et les possibilités de nouveaux matériaux. L'ascenseur de Santa Justa à Lisbonne, conçu par l'ingénieur Raoul Mesnier de Ponsard en 1900 et inauguré l'année suivante, constitue un exemple concret de travail paradigmatique de ce nouveau siècle: en utilisant la puissance d'un moteur à vapeur, cette œuvre célébrait les utopies urbaines matérialisées à travers les innovations techniques, établissant une communication entre le quartier de la Baixa et du Carmo et en se proposant comme élément novateur de la ville et du progrès accompli ${ }^{2}$. Dans cette nouvelle réalité industrielle, une fois de plus, le béton armé trouvera un bon terrain d'essai.

La première période de diffusion du béton armé au Portugal sétend pendant environ 30 ans depuis le début des années 90 du XIX ${ }^{e}$ siècle. Plusieurs références délimitent cette période: 1892 (avril) coïncide avec l'attribution du premier brevet Cottancin au Portugal à travers la personne de José Martins ; 1894 (septembre), date l'enregistrement du premier brevet Hennebique au Portugal à travers la personne de Jacques Monet; 1897, voit la construction de la Fábrica de Moagem de Caramujo; 1925 est l'année du début des travaux du Cinéma Capitolio, premier manifeste moderniste de l'architecte Luis Cristino da Silva; 1928 est la date de construction d'un entrepôt de trois étages à Santa Apolónia, conçu par João Jorge Coutinho avec une structure entièrement réalisée en béton armé, une superficie de mille mètres carrés et un toit à terrasse.

En 1898, le nouveau matériau, qui en moins de deux décennies remplaçait complètement le fer et la maçonnerie dans les constructions industrielles, a été adopté pour la première fois dans la construction d'un grand ouvrage public avec l'utilisation du brevet Cottancin : il s'agissait de l'édifice de la Escola Médica de Lisboa où le nouveau matériau était utilisé pour la mise en œuvre du plafond de l'atrium de l'édifice. On a connaissance de l'application de ce système grâce à un article publié, en 1899, dans la revue

\footnotetext{
2 Pour plus d'informations sur le sujet voir Santana, Sucena 1994.
} 
Revista de Obras Publicas e Minas de l'Associação dos Engenheiros Civis Portugueses (Meneses 1899: 40-47). Dans cet article Pedro Arnaut de Meneses, avec la collaboration de Cândido Xavier Cordeiro et Luciano de Carvalho, se prononçait sur les premières expériences en béton armé faites au Portugal; spécifiquement, il mentionnait la construction de ce plancher en ciment anglais Eagle, réalisé pour la Faculté de médicine de Lisbonne au champ de Santa Ana, dont les travaux furent dirigés par M. S. Reynaud \& C, à l'époque représentants au Portugal du brevet Cottancin.

Cela montre comment, dans ce temps là, par plusieurs entrepreneurs/ constructeurs du béton, le Portugal était vu comme un terrain vierge où expérimenter leurs propres inventions ou brevets dans le but de conquérir un marché encore pauvre de concurrence. Du moins, c'était avec cette conviction que François Hennebique, leader incontesté du béton armé dans de nombreux autres pays européens, en 1894 commençait à tourner son regard vers le Portugal, cherchant de convaincre l'entrepreneur belge Jacques Monet, qui depuis quelques années vivait à Lisbonne, de devenir son premier concessionnaire dans le pays:

les grands prix du fer à Lisbonne comme dans tout le Portugal doublent les avantages de mon système $[\ldots]$ le principe des douanes espagnoles et portugaises c'est celui de faire payer très cher les fers (profilés) et de favoriser l'entrée des fer bruts. Nous navons que l'emploi de barres sortant de laminoirs et de fers feuillards pour lesquels le travail de découpage et de pliage est très facile. Quant à la question des ciments des chaux hydrauliques, avec de bons sables et graviers on peut partout remplacer les ciments Portland pour les bétons [... ] Dans un pays comme le Portugal, alors, un magasin de 25 à 50 tonnes de barres vous permet d'entreprendre à l'instant tous les gîtages (Delhumeau 1999: 61-62).

En réalité on doit reconnaître que l'histoire de la diffusion du béton armé au Portugal est aussi bien liée au début de la production de Portland dans le pays; production réalisée initialement par une industrie de ciment localisée en Alhandra et de propriété de António Teófilo de Araújo Rato qui à partir de 1894 commençait la fabrication du matériau après avoir déposé son brevet pour la fabrication de ciment artificiel Portland (Ferreira 1989: 70). Le ciment "Tejo" produit dans cette usine fut le premier ciment Portland artificiel produit en Portugal. Initialement il fut obtenu par voie humide, à travers le four Hoffman, puis avec le four Candlot et après 1913 il fut produit avec le four Candlot-Perpignani par voie sèche.

A cette époque, alors, les conditions économiques et sociales étaient idéales pour se lancer dans de telles innovations technologiques. La production 
nationale de ce secteur était vu par le même António Rato comme nécessaire au développement général de tout le pays qui, si faisant, aurait pu montrer sa volonté de croissance industrielle et économique en permettant la réduction de l'importation de Portland d'autres pays étrangers comme la France, l'Allemagne, l’Angleterre. Ainsi expliquera António Rato cette conviction:

A fabricação de cimento artificial é entre nós completamente desconhecida [...]. Alguma produção de cimento se faz em Portugal, mas simplesmente de cimento natural e ainda assim em pequena escala, sendo esse producto consumido nos logares próximos a essa produção e os processos que se empregam n'esse fabrico são ainda os primitivos, limitando-se a cozedura e moagem dos calcários. N'estas condições não pode a indústria nacional, affrontar a concorrência do producto similar estrangeiro [...]. Estabelecer uma grande fábrica de cimento artificial, que possa pela sua qualidade e pelo seu custo módico fazer cessar, senão no todo pelo menos em parte, a importação estrangeira d'este artigo, foi o problema que me propus resolver (Ferreira 1989: 70-71).

Dans cette situation de croissance globale qui touchait le pays au tournant du siècle, Hennebique présentait son brevet à partir de 1894, année où il commençait à enregistrer son permis au ministère des Travaux Publics, du Commerce et de l'Industrie de Lisbonne. Le processus de brevetage d'une "invenção de um vigamento ligeiro e de grande resistência, em beton de cimento, com barras de ferro e estribos travessas embebidos em massa" fut lancé à Lisbonne, à travers une Exposition d'Inventions, le 13 octobre 1894 par Jacques Monet, auquel François Hennebique avait donné procuration de son brevet le 26 septembre de la même année. Cette exposition sera très importante pour la diffusion du brevet Hennebique au Portugal car fut caractérisée par deux revendications importantes: la première relative à la construction des poutres droites et courbes; la seconde, plus

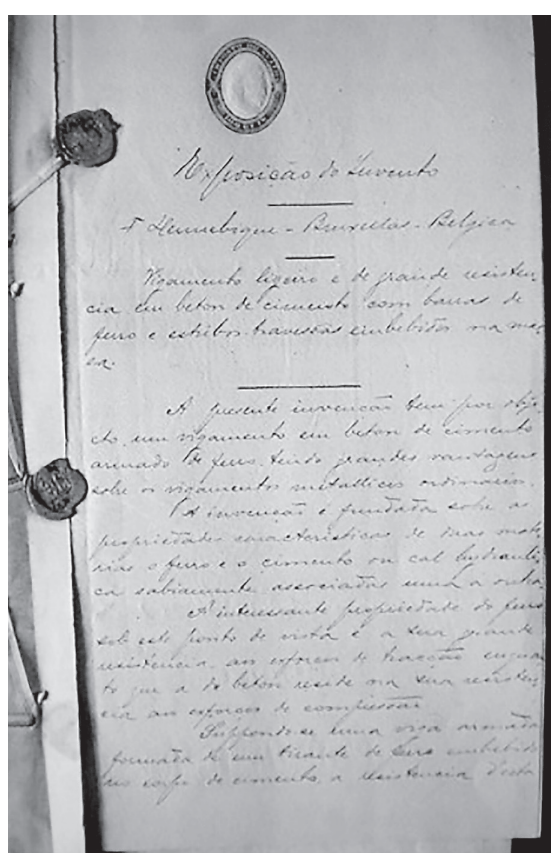

Fig. 3. Document de l'Exposição do Invento de 1894 où vient expliqué le brevet Hennebique (Ferreira 1989: 106) 
spécifique, relative à l'innovation clé du système Hennebique qui consistait dans l'utilisation de l'étrier, barre transversale d'acier destinée à reprendre l'effort tranchant. L'étrier, qui avait donc la tâche de lutter contre les efforts de cisaillement présents dans l'élément linéaire infléchi, deviendra bientôt le

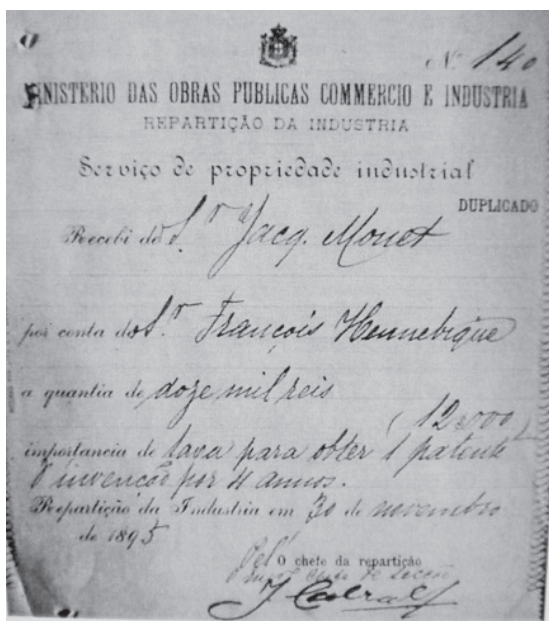

Fig. 4. Réception de l'impôt payé par Monet pour la licence du brevet Hennebique au Portugal pendant 4 ans. 30 novembre 1895 (Ferreira 1989: 110) symbole de la firme Hennebique, en lui donnant une longueur d'avance sur les concurrents.

Le 30 novembre 1895, au Ministère de Travaux Publics, du Commerce et de l'Industrie de Lisbonne, à trois heures et demie de l'après-midi, Jacques Monet obtient définitivement la reconnaissance du brevet et, ensemble, la possibilité de l'exploiter pour quatre ans en tant que procurateur d'Hennebique à Lisbonne. Moins de deux mois après la date de réception de la demande, la reconnaissance du brevet d'Hennebique serait ainsi révélée dans le Boletim da Propriedade Industrial de Lisbonne:

$\mathrm{N}^{\circ} 2108$ - Classe XII - François Hennebique, de Bruxelas, representado por J. N. Monet, morador em Verviers, Bélgica, por procuração substabelecido a Jacq Monet, residente em Lisboa, requereu no dia 30 de novembre de 1895, pelas três horas e meia da tarde, patente de invenção, por quatro anos, para um vigamento ligeiro e de grande resistência, de formigão (betão) de cimento, com barras de ferro e estribos travessas colocadas na massa (Ferreira 1989: 106).

Cette concession provisoire du brevet d'invention fut donnée le 20 juin 1896 , presque six mois après la date de demande de son homologation, qui avait eu lieu en novembre 1895, et révèle l'intérêt marqué que le constructeur et entrepreneur belge avait pour l'implantation de son système dans le nouveau marché portugais. Intérêt bien manifesté aussi à travers les pages de sa revue Le Béton armé, qui déjà en 1899 décrivait avec beaucoup de détails le premier travail important fait à Lisbonne par la Société Monet \& Filho: la reconstruction de la Fábrica de Moagem de Caramujo de José Antonio Gomes à l'Almada (Le Béton armé 1899, 14). Cette usine, complètement détruite après un incendie et ensuite rebâtie en béton armé, peut être considérée 
comme le premier bâtiment au Portugal à être entièrement construit en béton armé/brevet Hennebique. Larticle que Paul Christophe va publier dans la déjà mentionnée revue confirme non seulement le rôle important de l'entreprise Monet dans la construction de l'usine de Caramujo, mais permet également de corroborer certaines caractéristiques techniques de l'immeuble et aussi certains choix structurels réalisés directement par le bureau d'Hennebique à Paris. Dans l'article, Christophe décrit trois éléments fondamentaux de l'usine portugaise: la structure de l'ossature en béton, la façade et le toit . L'ensemble est décrit et commenté en reproduisant le projet de l'usine avec les plans d'étage, les dessins détaillés, la conception des perspectives architecturales de toute l'usine et le plan de la terrasse principale (Le Béton armé 1899, 14; Santos 1996: 295).

Alors, comme cela a été le cas dans certains autres pays où le brevet s'était propagé, comme par exemple l'Espagne, au Portugal les premiers bâtiments entièrement réalisés avec la nouvelle technique constructive étaient pour la plupart des établissements industriels et plus spécifiquement des usines de farine.

\section{La "Fábrica de Moagem de Caramujo".}

La reconstruction de l'usine de Caramujo est importante pour deux raisons spécifiques: en premier lieu parce qu'elle fut l'occasion pour le Portugal de s'expérimenter dans la construction de la première structure entièrement réalisée en béton armé avec des proportions aussi importantes, qui, maintenant, reste comme témoignage quasi unique d'un patrimoine presque perdu, et en second lieu, parce qu'elle représente pour le pays, à cette époque-là, un exemple unique d'unité structurelle soigneusement modulée et réalisée avec le système Hennebique. Les choix constructifs pris en compte pour la reconstruction du bâtiment en 1897 - colonnes, poutres et dalles pleines seront l'exemple le plus important et pionnier d'un pays qui à travers ce bâtiment voulait démontrer soit sa capacité d'exploitation du nouveau matériau soit sa croissance technologique liée à l'industrie.

La fabrique de Manuel José Gomes, fondée en 1865, commence une phase importante dans l'industrie meunière portugaise. Dans la dernière décennie des années quatre-vingt du $\mathrm{XIX}^{\mathrm{e}}$ siècle, cette usine avait connu un développement technologique très fort souligné par l'introduction de la machine à vapeur dans la production de grain, et par l'utilisation d'ouvriers qualifiés qui avaient amélioré la production. Au fil des ans, l’importance de 


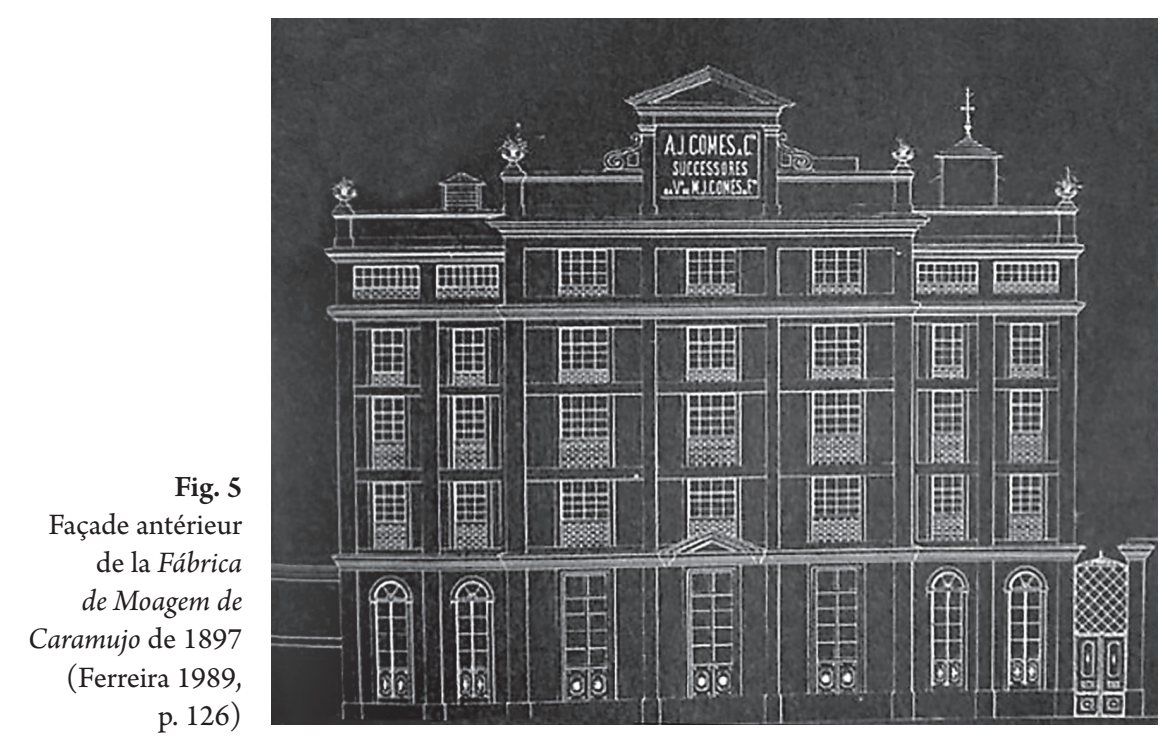

l'usine avait considérablement augmentée, comme en témoigne la présence d'un important ensemble structural de services industriels liés à l'usine, aujourd'hui toujours existants, qui à l'époque était l'un des plus importants au niveau régional.

Le 10 juin 1897, quand déjà l'usine était dirigée par le fils de Manuel Gomes, José António, un violent incendie détruit complètement l'établissement industriel. A la suite de cela, l'adoption du système Hennebique, qui propre dans ces années était salué comme le solutionneur le plus efficace contre les problèmes d'incendie, devient la meilleure solution (Segurado 1923: 6). Le nouveau bâtiment, construit en 1897 à la place de l'ancienne usine, était un édifice à forme trapézoïdale avec les côtés longs mesurant environ trente mètres et les plus petits côtés mesurant respectivement vingt-huit mètres et demi et vingt-cinq mètres et demi. Les longs côtés du trapèze correspondaient à la façade principale et à la façade postérieure, tandis que les petits côtés représentaient les façades latérales. Au rez-de-chaussée et au premier étage, la planimétrie du projet révélait l'existence de deux zones distinctes: une, contigüe à la façade principale, orientée à l'ouest avec une double hauteur et une grande zone structurée sans appuis intermédiaires; l’autre, postérieure et plus grande, structurée de façon modulaire avec des piliers de section carrée qui soutenaient des travées et des panneaux de dalles de dimensions d'environ trois mètres sur trois mètres et trente-cinq mètres sur trois mètres.

La structure du bâtiment reflétait le schéma du système Hennebique. Les dalles de plancher, d'une épaisseur de $12 \mathrm{~cm}$, étaient préparées pour supporter 


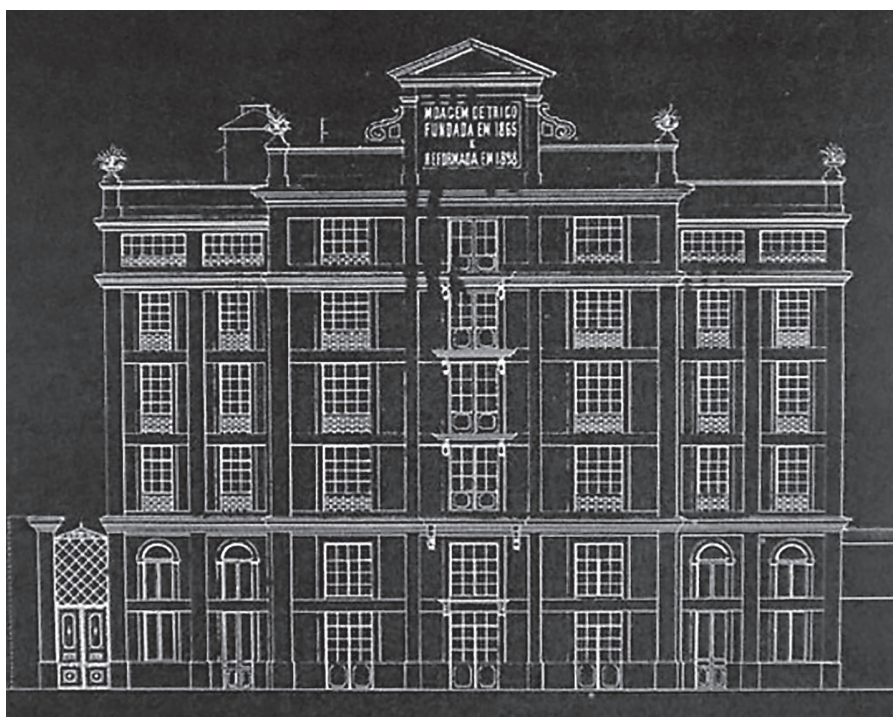

Fig. 6.

Façade postérieur de la Fábrica de Moagem de Caramujo de 1897 (Ferreira 1989: 65)

une charge de $1200 \mathrm{~kg}$ par mètre carré. Elles étaient armées de barres d'acier longitudinales de $12 \mathrm{~mm}$ de diamètre et d'étriers de profil $20 \mathrm{~mm}$ et de diamètre de $2 \mathrm{~mm}$. Les poutres présentaient les mêmes éléments, avec la différence que les barres d'acier, au lieu d'être placées côte à côte, étaient superposées et encadrées avec des étriers. Les poutres principales, orientées nord/sud, avaient les dimensions de $20 \mathrm{~cm}$ de largeur et de $30 \mathrm{~cm}$ de hauteur avec une variation de longueur qui allait de 3,50 m à 5,35 m (Le Béton armé 1899, 14; Santos 1996). Cela impliquait la présence de supports intermédiaires constitués par des poutres secondaires disposées est/ouest, qui, dans la construction de Caramujo, étaient positionnées à une distance d'environ 1,75 $\mathrm{m}$ les unes des autres. Il s'agissait d'une structure innovatrice pour l'époque, au point où le propriétaire António Gomes, en vue de tester la sécurité et la stabilité de l'édifice, posait sur le sol du bâtiment des sacs de sable très lourds pendant que la construction progressait (Flores 1992: 37). C'est ainsi que Paul Christophe décrivait le système utilisé pour les dalles de plancher de l'usine :

Nous donnons dans la planche XXXVIII, comme exemple de construction industrielle, les détails du plancher du I ${ }^{\text {er }}$ étage de la Minoterie Gomes, à Caramoujo (Caramujo) près Lisbonne (1897). La surface à couvrir est divisée par des poteaux en rectangles offrant aux poutres principales des portées de $3^{\mathrm{m}} 50,3^{\mathrm{m}} 75$ et $5^{\mathrm{m}} 35$, tandis que les poutres secondaires couvrent 3 mètres ou $3^{\mathrm{m}} 83$ avec un espacement de $1^{\mathrm{m}} 74$ à $1^{\mathrm{m}} 78$. Ce plancher est calculé pour une surcharge de 1,200 kilogrammes par mètre carré. Le hourdis n'est armé que dans un sens ( $L e$ Béton armé 1899, 16: 6.). 


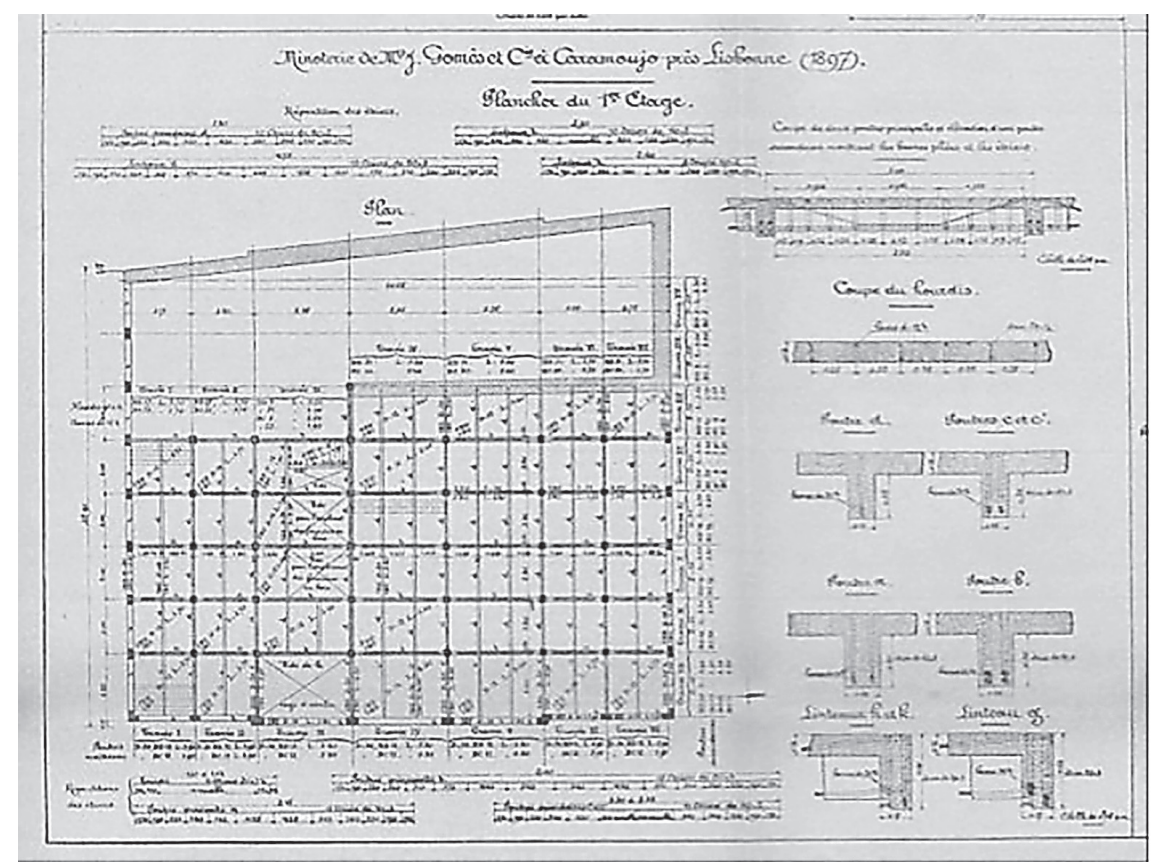

Fig. 7. Minoterie de M. M. Gomes et $C^{\mathrm{ie}}$ à Caramoujo près Lisbonne. Plancher du $1^{\mathrm{er}}$ étage, in Le Béton Armé 1899, 17: 5.

Fig. 8. José Maria da Silva, Fábrica de Moagem de Caramujo. Vue de l'édifice en construction, 1897 (Fonds Bétons Armés Hennebique, $\mathrm{BAH}$, Ifa $\mathrm{n}^{\circ}$ 076)

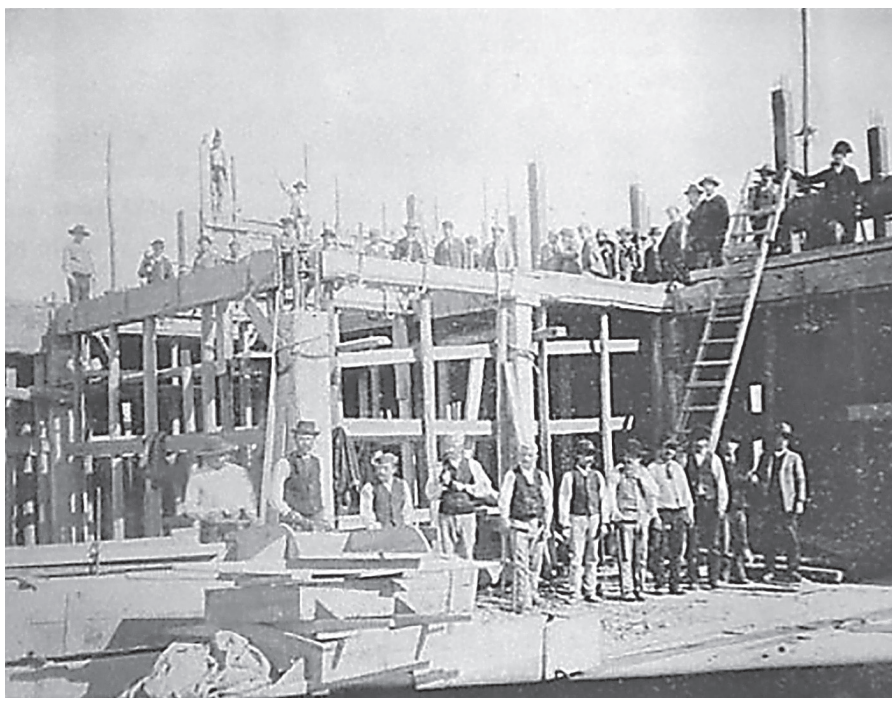


Dans la disposition générale du nouvel édifice, l'espace qui existait auparavant a été maintenu. Ainsi le schéma de base de l'organisation du bâtiment restait inchangé. Ils ont réalisé le même nombre d'étages que l'ancien bâtiment, à savoir six qui correspondent aux différentes phases de transformation des céréales. Ils ont également adopté la même séquence de production qui commençait avec la trituration des céréales au sommet de l'immeuble et se terminait avec la mise en sac au rez-de-chaussée. Au premier et au deuxième étage, on avait la salle des machines qui était considérée comme la force motrice de l'unité de production de l'immeuble. À cette époque, les deux machines à vapeur qui caractérisaient la fabrique s'y étaient installées et plus tard, avec l'avènement de l'électricité, elles ont été remplacées par des machines électriques.

Même la toiture, utilisée comme terrasse, a été une véritable innovation. Elle était munie d'un réservoir d'eau de $20 \mathrm{~m}^{3}$ qui pourrait servir en cas d'incendie. Ainsi, Paul Christophe a encore décrit cette solution étonnante sur les pages de la revue Le Béton Armé :

La planche $\mathrm{L}$ donne les détails d'un réservoir rectangulaire de 20 mètres cubes monté sur piliers en béton armé de 4 mètres de hauteur. Cette construction est établie sur la terrasse de la Minoterie Gomes à Caramoujo près de Lisbonne (1897). Le fond est plat. Il est muni de poutres secondaires et porte sur un cadre de poutres principales reposant sur les quatre piliers, les parois latérales de $1^{\mathrm{m}} 20$ de hauteur, ont $0^{\mathrm{m}} 13$ d'épaisseur maxima. Elles sont armées pour travailler comme consoles encastrées sur le plancher, c'est à dire que l'ossature, formée de tiges verticales, est voisine de la face intérieure sur laquelle la pression s'exerce. Suivant le bord supérieur, la paroi est cependant renforcée en forme de corniche et armé d'une barre horizontale qui donne un certain appui. Larmature des parois est retournée de $0^{\mathrm{m}} 70$ dans le hourdis du fond (Le Béton armé 1900, 21:5)3.

Même l'ingénieur J. M. d'Oliveira Simões, en $1899^{4}$, décrivait l'usine en ces termes de modernité et d'innovation technique:

É a mais moderna das fábricas de moagem em Portugal e uma das mais notávais. Para em tudo ser digna d'exame aos que se interessam pelas causas industriais e pela ciência do constructor, basta saber-se que, montada recentemente, em 1898, está dotada dos mais perfeitos mecanismos d'esta especialidade que

\footnotetext{
Voir aussi le no 17 de 1899, où, à la page 5, on fait mention à l'usine de Caramujo pour ce qui concerne la structure des façades.

4 Cf. Gazeta dos Caminhos de Ferro, n. 288, 1899.
} 
tanto se tem desenvolvido, e que a construção do edifício é o primeiro exemplar de valor da aplicação do novo processo de construção em cimento armado, systema Hennebique. O incêndio de 10 de Junho de 1897 deu ocasião a que se fizesse uma transformação radical na antiga fábrica. Diversas transformações tinha experimentado já durante a evolução do seu trabalho, sendo a mais importante realizada em 1869, mas nunca se fizera uma transformação tão completa. $\mathrm{O}$ conjunto tem um aspecto gracioso e arquitectónico, acomodado ao fim que se teve em vista (Santos 1996: 88-89).

Euvre pionnière d'un matériau et exemple symbolique d'une architecture industrielle moderne, dans le sens d'une expression moderne des formes, l'usine de Caramujo doit être aussi considérée comme une référence importante dans le panorama international. Par exemple, si on la compare au niveau structurel à certains travaux similaires, on peut reconnaître les paramètres techniques et architecturaux présents dans les différentes constructions d'Hennebique. La "Filature de Charles Six" à Tourcoing, le "Moulin de blé" de Nantes et l'usine textile des frères Barrois à Lille, tous les trois construits entre 1895 et 1896, constituent des exemples probants de ce système.

Il semble aussi approprier de comparer l'usine de Caramujo avec d'autres immeubles similaires réalisés par Hennebique à la même époque: comme le "Moulin Idéal" (dans la Loire) et le "Weaver's Mill" (Swansea, Royaume-Uni), tous les deux construits en 1898. Ce dernier en particulier, conçu par l'architecte Henry Portsmouth en 1897, a la particularité d'être retenu comme le premier bâtiment en béton armé de son pays, de même que l'usine

Fig. 9. José Maria da Silva, Fábrica de Moagem de Caramujo. Bâtiment terminé, 1898 (Fonds Bétons Armés Hennebique, $\mathrm{BAH}$, Ifa $\mathrm{n}^{\circ}$ 076)

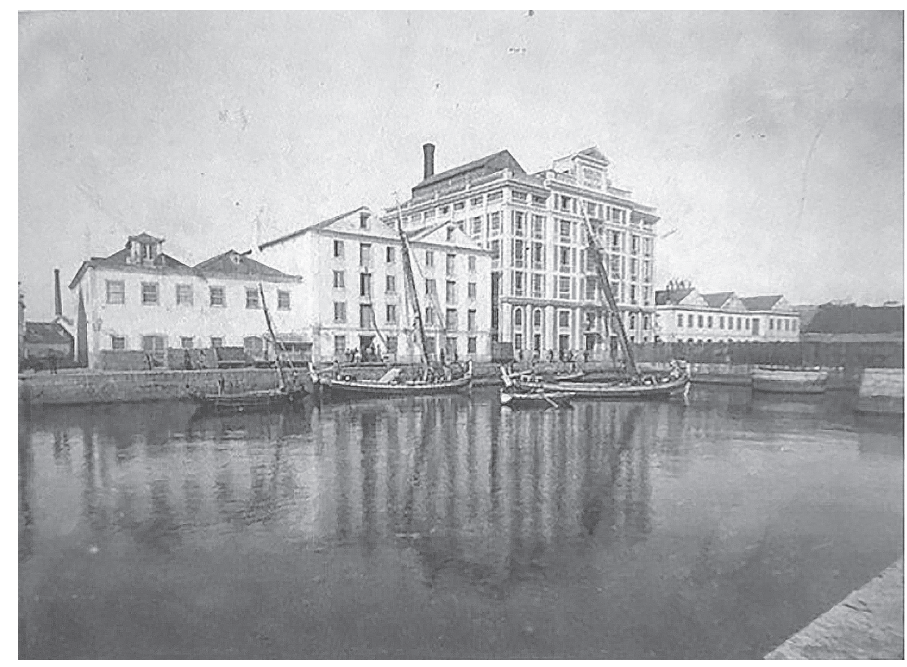


portugaise. Il se compose d'un bloc solide destiné aux silos et d'une usine de fraisage avec sept étages, dont les dimensions et la répartition des vides et des pleins permet d'établir une certaine parenté avec l'organisation de Caramujo (Santos 1996: 88-89). Plus tard, il y aura aussi le complexe du moulin "William Vernon \& Fils" (à Londres), conçu par l'architecte John Clarke, et surtout la coopérative "Wholesale Society", construite entre 1903 et 1908, où l'on trouve la même conception de façade quà l'usine de Caramujo, surtout en ce qui concerne la modulation et la distribution des ouvertures ( Le Béton Armé 1899, 12: 5-6; 17: 202-205). Cela est strictement lié à l'idée de "plan et façade libres" rendu possible grâce au système Hennebique.

\section{L'héritage de l'usine de Caramujo dans les bâtiments portugais des années à venir}

Après l'exemple de l'usine de Caramujo, et pour la connaissance de l'emploi du béton armé au Portugal, de nombreux d'autres cas peuvent être mentionnés comme également importants. On connaît en fait que Monet \& Fils resteront actifs dans le Pays au moins jusqu’au début de 1900, quand ils réaliseront la version finale de la nouvelle usine de tissage de la "Société Grandella \& C.", à São Domingos de Benfica (Santos 1996: 297). De plus, en février 1904, déjà sans son représentant Monet, Hennebique présentera à travers un autre agent officiel, un certain Machado da Cruz, une demande d'un nouveau brevet pour la fabrication des tuyaux, des canaux ou des colonnes de béton, ou d'autre aggloméré, armé ou non ${ }^{5}$. Immédiatement après, et précisément le 30 avril, il sera enregistré au Portugal le premier brevet en béton préfabriqué pour des panneaux de plancher et des poutres; tout à fait en simultané à l'application, dans le Pays, de piliers de fondation pour les travaux maritimes. En novembre 1905, une déclaration publiée sur la revue $A$ Construção Moderna, fait connaître le nom de la nouvelle Société chargée de l'exploitation du brevet Hennebique au Portugal: la Société Moreira de Sá \& Malevez (Quintela 1990: 9-15). À l'époque, cette entreprise avait déjà commencé à s'occuper de la construction de grands ouvrages en béton armé, soit en Espagne soit au Portugal. Déjà en 1902, en fait, l'un des partenaires de la société future, l'ingénieur Bernardo Joaquim Moreira de Sá, associé à la mai-

\footnotetext{
Patente no 4560, demandée le 22/2/1904 et donnée pour deux années à partir de 9/6/1903 dans les termes de l'art. 29 de la carta de lei de 21/5/1896. Cf. Boletim da Propriedade Industrial, Lisboa, $3^{\text {e }}$ série, 21 (3), mars 1904, p. 69; et 21 (7), juillet 1904, p. 194.
} 
son Hennebique de Madrid, avait réalisé des premiers travaux dans la ville de Porto comme concessionnaire du brevet. Ensuite, en octobre 1903, cette fois en tant que responsable à Porto de la nouvelle société de construction qui portait son nom, il avait commencé la réalisation de la seconde œuvre en béton armé du Portugal : il s'agissait de la Caserne de Pompiers commissionnée par la municipalité de la ville. Un immeuble de quatre étages, avec une hauteur totale de 19 mètres sur d'une superficie de $65 \mathrm{~m}^{2}$, qui était caractérisé par la présence d'un plan libre dans l'organisation de l'espace, nécessaire à la corporation des pompiers pour la réalisation quotidienne de leurs exercices de préparation (Quintela 1990: 9-15). Ainsi l'entreprise Moreira de Sà décrivait les circonstances qui avaient amené à la naissance de cette œuvre architecturale et le choix du béton armé en tant que matériau du projet:

O município do Porto possuía há vários anos um terreno vasto, em declive, de aterro muito heterogéneo e em que o solo bom se encontrava a $18 \mathrm{~m}$ de profundidade. $\mathrm{O}$ município pensava ocupar este terreno com o novo quartel dos bombeiros, mas o alto custo que implicaria a fundação num tal terreno impedia-o de tomar uma decisão. Propusemos então construir em Betão armado os diversos edifícios, incluindo as fundações. Seria a primeira obra de Betão armado executada em Portugal e, justamente por isso, foi necessário um pouco de trabalho para convencer o município. [...] a natureza do solo que deveria suportar o edifício era [...] o que constituía o ponto mais difícil a resolver [...]. A adopção do Betão armado impunha-se do ponto de vista económico e dava inteira e plena satisfação no aspecto técnico. [...] o edifício [...] tem $6,50 \mathrm{~m}$ de fachadas e $10 \mathrm{~m}$ de comprimento e uma pequena torre, sobre a esquerda, de 2,70 $\mathrm{m}$ de lado, sobre uma laje geral de Betão armado que distribui a carga total na razão de $500 \mathrm{grf} \mathrm{cm} 2$. Esta laje tem $10 \mathrm{~cm}$ de espessura, mas sob cada pilar tem $2 \mathrm{~m} \times 2 \mathrm{~m}$ e $30 \mathrm{~cm}$ de altura. Os pilares, lintéis, pavimentos, vigas e cobertura são de Betão armado; as paredes foram feitas com tijolos colocados com $11 \mathrm{~cm}$ de espessura e selados aos pilares simplesmente por meio de cimento. Os pavimentos assim como a cobertura tem $8 \mathrm{~cm}$ de espessura (Quintela 1990: 12).

Même si dans cette description, à tort, on parle de cet ouvrage comme le premier projet entièrement réalisé en béton armé (on ne doit pas oublier, en fait, la construction de l'usine de Caramujo réalisée quelque année auparavant), elle est, malgré tout, très intéressante pour bien comprendre les raisons qui parfois étaient arrière le choix de la nouvelle technique de construction: l'économicité et l'extraordinaire capacité portante.

Ensuite, en 1904, cette entreprise va réaliser le premier pont en béton armé du pays, à savoir l'élégant pont de "Vale de Meões", à Mirandela, qui 
avec 19 mètres de longueur sera construit en seulement trente quatre jours. A partir de 1906, on aura la réalisation d'autres ouvrages importants: un viaduc en Arraiolos (1906) et deux ponts de plus larges proportions faits respectivement à Oliveira de Frades, Ponte Luiz Bandeira de 44 mètres de longueur pour un arc d'ouverture de 32 mètres (1907), et à Braga, Ponte de Parada de 33 mètres de longueur (1908) (Santos 1996: 181-184; Revista de Obras Públicas e Minas 1908, 39: 25).

En 1911, on a le projet d'un dépôt de wagons à construire à Campolide (Lisbonne) pour la Société de Wagons-Lit. Alors que, en 1912, le béton armé est utilisé dans la construction du premier dépôt d'eau du Portugal, construit à Entroncamento par la "Société des Chemins de Fer Portugais". Avec une capacité de $250 \mathrm{~m}^{3}$, il s'agissait d'une construction caractérisée par une géométrie rigoureuse et une grande pureté formelle, où les bords structurels des traverses de contreventement et des minces piliers qui prenaient en charge le réservoir étaient modelés doucement pour rendre la structure la plus raffinée possible. Petite finesse architecturale appliquée à une infrastructure industrielle. En 1913, d'autres ponts et viaducs, entièrement en béton armé, ont été construits par cette Société; tandis que, comme exemple d'un stade initial d'intégration du nouveau matériau dans les techniques constructives traditionnelles, qui étaient toujours utilisées, on peut mentionner, à Lisbonne, la construction de la Fábrica de Cerveja Portugália de António Rodrigues da Silva Júnior, construite à partir de 1913 par la Société Fernand Touzet (Ferreira 1972: 22). De la même époque, on a la construction d'un pont encore plus important que celui de Meões, le Pont de Alvor, ayant comme principaux éléments de résistance deux arcs jumelés de 33,60 mètres d'ampleur, contreventés par des poutres.

Autour des années vingt du $\mathrm{XX}^{\mathrm{e}}$ siècle le béton armé est désormais un élément propre de l'architecture portugaise. Mentionnons à ce propos le projet présenté en 1928 par l'ingénieur João Jorge Coutinho pour la construction d'un entrepôt à Lisbonne. Il s'agissait d'un bâtiment construit à Santa Apolónia et entièrement réalisé en béton armé. De forme rectangulaire, avec trois étages, un toit à terrasse et une superficie de mille mètres carrés, ce bâtiment déjà à la fin des années vingt était salué par le monde architectural portugais avec des commentaires critiques très élogieux. À rendre encore plus évidente l'importance architectonique du béton armé, ce sera enfin le Mouvement Moderne qui, depuis la moitié des années vingt du XX siècle, commencera à se diffuser aussi dans la réalité constructive portugaise. Ensuite, les années trente et quarante offrent dans le panorama portugais la preuve d'une véritable acceptation du matériau qui vient, en ce moment là, 
encore plus reconnu pour ses remarquables capacités structurales et pour son potentiel formel. On pense, par exemple, à l'Église de Notre-Dame de Fátima à Lisbonne (1938), qui avec son architecture va séloigner définitivement de la tradition constructive jusqualors considérée, en devenant l'un parmi les premiers bâtiments au Portugal où le béton armé vient utilisé aussi pour sa valeur esthétique.

De la même époque, ce sont d'autres exemples remarquables comme la Casa da Moeda e Valores Selados de Lisbonne (1930-1940), saluée comme l'une des œuvres les plus singulières du premier modernisme portugais (Tostões 2005: 66), qui se distingue des autres œuvres architecturales des années précédentes par son rationalisme constructif et son fonctionnalisme programmatique; ou encore le siège du journal Diário de Notícias (1936-1939), sur l'Avenida da Liberdade, qui résout de façon régulière et géométrique une façade sur rue compliquée; ou la Lota de Massarelos de Porto (1933-1935), également connue avec le nom de Bolsa do Pescado ou Entreposto Frigorífico do Peixe, où l'architecte Januário Godinho réalise son premier ouvrage de grande puissance expressive, en assemblant influences multiples, et où ils sont les exigences fonctionnelles qui déterminent la relation entre la spatialité interne et le système structural (Tostões 2005: 66).

À ces bâtiments on peut ajouter beaucoup d'autres également importants tels que la Fábrica Barros (1947-1952) et A Nacional de Lisbonne (19481956), l'Armazém Frigorifico de Lisbonne (1939-1944) et de Porto (19371939), la Tobis Portuguesa S.A. de Lisbonne (1936-1944), l'Algarve Exportador (1938) et la Fábrica Rainha do Sado (1941) à Matosinhos, la Portugália Fábricas de Malte e Cerveja de Lisbonne (1951), pour n’en citer que quelques-uns. Il s'agit de bâtiments qui bien expliquent comment, désormais, le béton armé était un matériau bien connu et bien utilisé par les architectes et les ingénieurs portugais. Ensuite, dans les années suivantes la contribution du matériau dans le monde constructif portugais sera encore plus prépondérante, en déterminant ce bouleversement de conception et ce changement de tendance qui se concrétisera dans la plupart des meilleures réalisations qui aujourd'hui illustrent le patrimoine architectural du pays. 


\section{Bibliographie}

\section{Sources d'archives}

"Fonds Bétons armés Hennebique" (BAH). Subdiv. 50: Autres pays - Avant 1903. Dossier 076 Ifa. Objet BAH-25-1897-09173. Minoterie Gomes et Cie, Caramujo, Lisbonne, Portugal. 1897. Cité de l'architecture \& du patrimoine /Centre d'archives d'architecture du XX $X^{\mathrm{e}}$ siècle (Ifa).

\section{Études}

APPLETON, Júlio (2016). Construções em betão - Nota histórica sobre a sua evolução (http:// www.civil.ist.utl.pt/ cristina/GDBAPE/ConstrucoesEmBetao.pdf, consulté en 2016.03.03).

DELHUMEAU, Gwenaël (1999). L'invention du Béton armé: Hennebique 1890-1914. Paris: Éditions Norma.

FERREIRA, Carlos Antero (1972). Betão aparente em Portugal. Lisboa: Associação Técnica da Indústria do Cimento.

FERREIRA, Carlos Antero (1989). Betão: a idade da descoberta. Lisboa: Ed. Passado Presente.

FLORES, Alexandre (1992). António José Gomes: o homem e o industrial. Cova da Piedade: Junta de Freguesia.

FARINHA, J. S. Brazão (1997). Construção da Baixa Pombalina. Lisboa: Metropolitano.

IORI, Tullia (2001). Il cemento armato in Italia: dalle origini alla seconda guerra mondiale. Roma: Edilstampa.

LICORDARI, Mariangela (2013). Le béton armé dans les bâtiments industriels: le cas de Brescia au début du $\mathrm{XX}^{\mathrm{e}}$ siècle. Paris: Université Paris1 Panthéon-Sorbonne (mémoire du Master Erasmus Mundus TPTI).

MENESES, Arnaut P. de (1899). "Experiencias na escola médica de Lisboa, e em França na escola de pontes e calçadas e no ministerio da guerra”, Revista de Obras Publicas e Minas, 30 (351-352), 40-47.

QUINTELA, António de Carvalho (1990). “Contribução para a história do betão armado em Portugal: primeiras obras”, Revista Portuguesa de Engenharia de Estruturas, 30, 9-15.

SANTANA, Francisco; Sucena, Eduardo (1994). Dicionário da história de Lisboa. Lisboa: Gráfica Europam.

SANTOS, António Maria (1996). Para o estudo da arquitectura industrial na região de Lisboa (1846-1918). Lisboa: FCSH-UNL (dissertação de mestrado policopiada).

SANTOS, Tito Nuno Sampaio Fernandes dos (2008). Estratégias para reparação e reforço de estruturas de betão armado. Braga: Universidade do Minho (dissertação de mestrado policopiada). 
SEGURADO, João Emílio dos Santos (1923). Cimento Armado. Lisboa: Tip. Empresa Diário de Noticias.

SIMMONET, Cyrille (2005). Le béton. Histoire d'un matériau. Marseille, Éd. Parenthèses.

TOSTÕES, Ana (2005). "Em direcção a uma estética industrial: zeitwill ou vontade de modernidade”, in A arquitectura da indústria, 1925-1965. Registo DOCOMOMO Ibérico. Barcelona: Fundação DOCOMOMO Ibérico.

VISEU, Joaquim C. S. (1993). História do betão armado em Portugal: incluindo a história do betão pré-esforçado. Técnicas relevantes. Obras conhecidas. Regulamentos principais. Lisboa: ATIC.

\section{Revues}

Boletim da Propriedade Industrial (1904), 21 (3), 21 (7).

Gazeta dos Caminhos de Ferro (1899) n. 288.

Le Béton armé, organe des concessionnaires et agents du système Hennebique (1898) 9; (1899) 12, 14, 16, 17; (1900) 1, 26, 28; (1901) 36, 37; (1902) 46, 49, 50 ; (1904) 69.

Revista Portuguesa de Engenharia de Estruturas (1990) 30.

Revista de Obras Públicas e Minas (1899) 30; (1908) 39. 